\title{
Erfolgreiche Behandlung eines Afatinib-refraktären nicht kleinzelligen Bronchialkarzinoms mit seltenen komplexen EGFR-Mutationen mit Pembrolizumab: ein Fallbericht
}

\author{
Yuri Taniguchi Momoko Yamamoto Hiroaki Ikushima Sayaka Ohara Hideyuki Takeshima \\ Toshio Sakatani Kazuhiro Usui
}

Division of Respirology, NTT Medical Center Tokyo, Tokio, Japan

Schlüsselwörter

Afatinib · seltene EGFR-Mutationen · Immuncheckpoint-

Inhibitoren · Nicht kleinzelliges Bronchialkarzinom .

Pembrolizumab

\section{Zusammenfassung}

Trotz der signifikanten Fortschritte, die in der jüngeren Vergangenheit in der Behandlung mit Immuncheckpoint-Inhibitoren (ICI) erzielt wurden, ist nach wie vor umstritten, ob sie auch bei der Behandlung des nicht kleinzelliges Bronchialkarzinoms (NSCLC) mit Mutationen des epidermalen Wachstumsfaktor-Rezeptors (EGFR) zum Einsatz kommen sollten. Wir berichten hier über den Fall einer NSCLC-Patientin mit seltenen, komplexen EGFR-Mutationen (G719S und L861Q), die gegenüber Afatinib refraktär war, auf Pembrolizumab jedoch ein gutes Ansprechen zeigte. Bei einer 65-jährigen ehemaligen Raucherin wurde ein NSCLC des rechten Oberlappens diagnostiziert (klinisches Stadium IVB; cT2bN3M1c). Unter einer

zweimonatigen Behandlung mit Afatinib war ihre Krankheit rapide weiter fortgeschritten. Die Behandlung mit Pembrolizumab wurde eingeleitet, weil mehr als 75\% ihrer Tumorzellen PD-L1 exprimierten. Ihr Tumor sprach gut auf die Pembrolizumab-Behandlung an, und die Wirkung hielt mehr als ein Jahr an. Dieser Fall deutet darauf hin, dass Pembrolizumab eine Behandlungsoption für NSCLC-Patienten mit seltenen EGFR-Mutationen und einem hohen Maß an PD-L1-Expression darstellen könnte, die auf eine EGFR-TKI-Therapie nicht ansprechen.

(C) 2019 Die Autoren. Herausgegeben von S. Karger AG, Basel

\section{Einleitung}

Die Entwicklung der Immuncheckpoint-Inhibitoren (ICI) hat in den letzten Jahren einen Durchbruch in der Behandlung des fortgeschrittenen nicht kleinzelligen Lungenkarzinoms (non-small cell lung carcinoma, NSCLC) gebracht. Pembrolizumab, ein humanisierter monoklonaler Antikörper, der PD-1 (programmed death 1) blockiert, ist heute die Standard-Erstlinientherapie, wenn bei fortgeschrittenem NSCLC über 50\% der Tumorzellen PD-L1positiv sind [1], und auch Pembrolizumab in Kombination mit einer platinhaltigen Chemotherapie hat sich als Option für die erste Therapielinie etabliert [2]. Trotz dieser wichtigen Fortschritte ist der Nutzen von ICI in der Behandlung des NSCLC mit Mutationen des EGFR (epidermal growth factor receptor) noch unklar. Bei NSCLC-Patienten mit sensibilisierenden EGFR-Mutationen sind ICI weniger wirksam, und die Behandlung mit EGFR-spezi-

\section{KARGER}

Fax +497614520714

information@karger.com

www.karger.com
() 2019 S. Karger GmbH, Freiburg

Accessible online at:

www.karger.com/kkp
Yuri Taniguchi

Division of Respirology, NTT Medical Center Tokyo

5-9-22 Higashigotanda

Shinagawa, Tokio 1410022, Japan

E-Mail yuriteito@gmail.com 

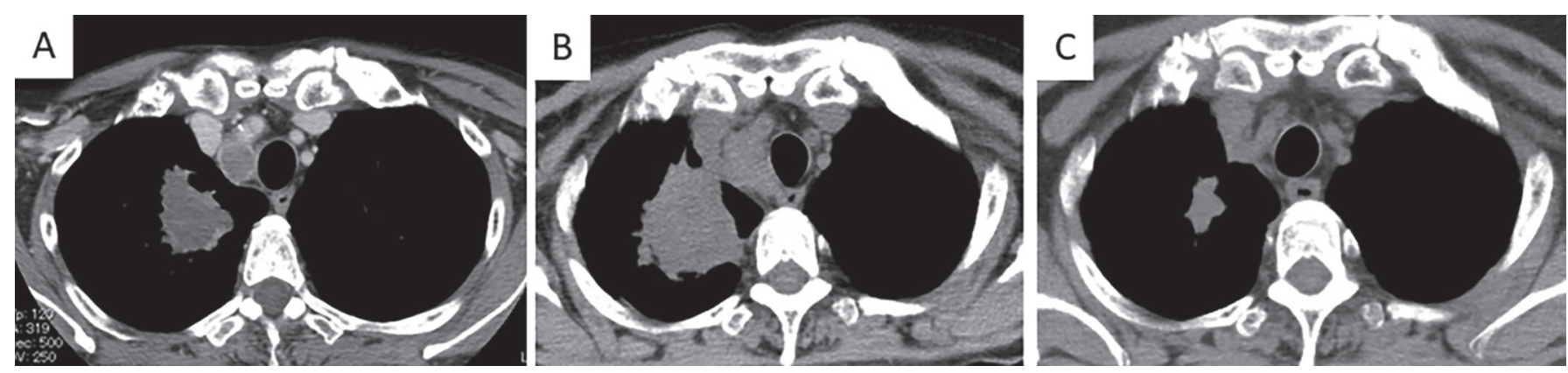

Abb. 1. Thorax-CT zum Zeitpunkt der Diagnose (a), 2 Monate nach der Afatinib-Behandlung (b) und nach einem Jahr Pembrolizumab-Behandlung (c).

fischen Tyrosinkinase-Inhibitoren (TKI) stellt den Therapiestandard dar. Doch nicht alle Patienten mit EGFR-Mutationen sprechen gut auf EGFR-TKI an. Seltenere EGFR-Mutationen, wie z.B. Substitutionen in Exon 18 (G719X, E790K/E790A), Insertionen und/oder Punktmutationen in Exon 20 (S768I), Insertionen in Exon 19 oder Mutationen in Exon 21 (L861Q) machen etwa 10\% der Gesamtheit der EGFR-Mutationen aus [3]. EGFR-TKI haben in der Behandlung von Krebserkrankungen mit diesen seltenen EGFR-Mutationen geringere Wirksamkeit gezeigt als bei Krebserkrankungen mit den häufigen Mutationen (Exon-19-Deletion und L858R). Angesichts dieses schlechten Ansprechens auf EGFR-TKI werden für Patienten mit seltenen EGFR-Mutationen andere Standardtherapieoptionen benötigt.

Wir berichten hier über den Fall einer Patientin mit NSCLC mit seltenen EGFR-Mutationen, bei der die Erstlinientherapie mit Afatinib versagte, die Zweitlinienbehandlung mit Pembrolizumab hingegen erfolgreich war.

\section{Fallbericht}

Eine 65-jährige ehemalige Raucherin stellte sich in der HNOAbteilung unseres Krankenhauses mit einem $1 \mathrm{~cm}$ großen Knoten auf der Zunge vor. Eine Feinnadelaspiration (FNA) dieses Zungentumors ergab nur atypische Zellen. Sie wurde an unsere Abteilung überwiesen, nachdem ein CT (Computertomographie)-Scan einen solitären Tumor am rechten Oberlappen der Lunge ergeben hatte (Abb. 1a). In der Bronchoskopie zeigte sich eine Obstruktion des rechten B1 durch einen Tumor, der als nicht kleinzelliges Karzinom diagnostiziert wurde. In der immunhistochemischen Färbung erwies sich der Tumor als negativ für TTF-1/p40/CK7; in der PNA-LNA-PCR-Clamp (petide nucleic acid-locked nucleic acid polymerase chain reaction clamp)Methode wurden EGFR-Mutationen in Exon 18 (G719S) und Exon 21 (L861Q) nachgewiesen. In der immunhistochemischen Färbung auf PD-L1 (IHC 22C3) waren über 75\% der Tumorzellen positiv gefärbt. Anhand der PET-CT (Positronenemissionstomographie-Comutertomographie)-Bildgebung wurde der Verdacht auf Metastasenbefall der rechtsseitigen mediastinalen, hilären und subclavialen Lymphknoten sowie der linken Nebenniere erhoben, und die Kopf-Magnetresonanztomographie (MRT) ergab eine metastatische Läsion von 4,5 mm Durchmesser am rechten Frontallappen. Auf der Grundlage dieser Befun- de wurde die Diagnose cT2bN3M1c (ADR, BRA, LYM, OTH), Stadium IVB, gestellt.

Obwohl eine Behandlung mit Afatinib 30 mg/Tag eingeleitet wurde, progredierten sowohl das primäre Nest als auch die Lymphknotenmetastasen innerhalb von 2 Monaten (Abb. 1b). Wir setzten daraufhin das Afatinib ab und stellten die Patientin auf Pembrolizumab um. Die Patientin sprach gut auf die Pembrolizumab-Behandlung an, und die Wirkung hält nun seit mehr als einem Jahr an, ohne dass schwerwiegende Nebenwirkungen aufgetreten sind (Abb. 1c).

\section{Diskussion}

EGFR-Mutationen sind bei etwa 40-60\% der Menschen ostasiatischer und etwa 10\% der Menschen kaukasischer Abstammung nachzuweisen. 45-50\% bzw. 40-45\% dieser Mutationen entfallen auf die beiden häufigen, sensibilisierenden Mutationsformen, Exon-19-Deletionen und die L858R-Mutation von Exon 21, während einzelne oder seltene komplexe Mutationen 2,6-14\% aller Mutationen ausmachen [4]. Seit der randomisierten Phase-IIIStudie IPASS (Iressa Pan Asian Study) sind EGFR-TKI in der Erstlinientherapie des EGFR-mutanten NSCLC fest etabliert, zeigen jedoch bei Patienten mit selteneren EGFR-Mutationen tendenziell weniger Wirkung $[3,5]$. Afatinib, ein EGFR-TKI der zweiten Generation, gilt als wirksamer als die TKI der ersten Generation, weil es ein irreversibler Inhibitor ist, der die hohe Bindungsaffinität dieser Mutationen an ATP aufhebt [6]. Die hier vorgestellte Patientin, bei der seltene, komplexe EGFR-Mutationen vorlagen (G719S + L861Q), zeigte während der zweimonatigen AfatinibTherapie rapide Progression und kein Ansprechen.

Eine Subgruppenanalyse von 3 randomisierten Studien ergab, dass Immuncheckpoint-Inhibitoren in der Behandlung von NSCLC mit onkogenen Driver-Mutationen in der Regel weniger wirksam sind als Docetaxel [7, 8]. Wir dürfen jedoch bei der Behandlung von Patienten mit EGFR-Mutationen nicht vollständig auf ICI verzichten, denn bei einigen Patienten mit EGFR-Mutationen wurden mit PD-1-Inhibitoren lange Überlebenszeiten erzielt. So ergab eine kürzlich veröffentlichte Phase-III-Studie, dass Patienten mit EGFR-Mutationen, die Atezolizumab in Kombination mit einer Chemotherapie aus Carboplatin, Paclitaxel und Bevacizumab erhielten, ein besseres progressionsfreies Überleben (progression-free survival, PFS) zeigten als Patienten, die eine al- 
leinige Chemotherapie erhielten [9]. Diese einzigen beiden aktuellen Studien unterstreichen, dass seltene EGFR-Mutationen mit der Wirksamkeit von ICI gegen EGFR-mutantes NSCLC assoziiert sein könnten $[10,11]$. Yoshida et al. benannten in ihrem Bericht eine starkes Rauchen in der Vorgeschichte (Raucherindex >30 Packungsjahre), eine kürzere Ansprechdauer auf EGFR-TKI sowie das Vorliegen seltener EGFR-Mutationen als prognostische Faktoren im Hinblick auf die Wirksamkeit von Nivolumab bei NSCLC mit EGFR-Mutationen [10]. Yamada et al. kamen zu dem Schluss, dass seltene Mutationen, T790M ausgenommen, mit einem guten Ansprechen auf ICI assoziiert sind [11]. Ähnlich wie in früheren Fallberichten sprach auch unsere Patientin gut auf die Pembrolizumab-Behandlung an. Immuncheckpoint-Inhibitoren könnten somit eine Option für die Folgebehandlung von NSCLCPatienten mit seltenen EGFR-Mutationen sein, bei denen eine EGFR-TKI-Therapie versagt hat.

\section{Schlussfolgerung}

Wir berichteten über einen Fall von NSCLC mit seltenen, komplexen EGFR-Mutationen (G719S + L861Q), bei dem ein signifikantes Ansprechen auf eine Zweitlinientherapie mit Pembroli- zumab beobachtet wurde, nachdem eine Erstlinientherapie mit Afatinib versagt hatte. Pembrolizumab könnte eine Behandlungsoption für NSCLC-Patienten mit seltenen EGFR-Mutationen und einem hohen Maß an PD-L1-Expression sein, die auf eine EGFR-TKI-Therapie nicht ansprechen.

\section{Erklärung zu ethischen Konflikten}

Die Autoren haben keine ethische Konflikte offenzulegen. Von der Patientin wurde nach Aufklärung eine schriftliche Einverständniserklärung für die Veröffentlichung des vorliegenden Fallberichts eingeholt.

\section{Disclosure Statement}

Die Autoren haben keine Interessenskonflikte offenzulegen.

\section{Literatur}

Die Literatur ist als Supplemental Material unter www.karger. com/?DOI=503948 abrufbar. 